\title{
Polysèmes
}

Revue d'études intertextuelles et intermédiales

\section{Le Choc sémantique de la métaphore dans The Red Badge of Courage de Stephen Crane}

\section{Marie-Odile Salati}

\section{(2) OpenEdition}

\section{Journals}

\section{Édition électronique}

URL : http://journals.openedition.org/polysemes/1658

DOI : 10.4000/polysemes. 1658

ISSN : 2496-4212

Éditeur

SAIT

\section{Édition imprimée}

Date de publication : 1 janvier 2005

Pagination : 101-120

ISSN : 0999-4203

\section{Référence électronique}

Marie-Odile Salati, «Le Choc sémantique de la métaphore dans The Red Badge of Courage de Stephen Crane », Polysèmes [En ligne], 7 | 2005, mis en ligne le 10 janvier 2017, consulté le 01 mai 2019. URL : http://journals.openedition.org/polysemes/1658; DOI : 10.4000/polysemes.1658

Ce document a été généré automatiquement le 1 mai 2019.

Polysèmes 


\title{
Le Choc sémantique de la métaphore dans The Red Badge of Courage de Stephen Crane
}

\author{
Marie-Odile Salati
}

1 Dans une lettre écrite deux ans après la parution de The Red Badge of Courage, Joseph Conrad saluait en l'auteur de l'ouvrage l'authentique «impressionniste» qui avait su recréer « l'illusion de la réalité »" Venant du théoricien du « faire voir » énoncé peu après dans la préface de The Nigger of the Narcissus, cet éloge prend toute son importance quand on se souvient que le jeune romancier n'a jamais été le témoin de la guerre de Sécession évoquée dans ses pages trente ans après l'événement. Et pourtant, contrairement aux productions antérieures de vétérans fidèles à une tradition de littérature épique et patriotique, le roman de Stephen Crane parvient à suggérer le vécu d'un soldat ordinaire et la violence inouïe du champ de bataille que le héros découvre au travers de ses illusions naïves de jeune engagé.

2 Parmi les procédés mis en œuvre pour donner à voir et à entendre les combats fracassants, un en particulier attire l'attention par son usage répété et ses effets percutants : la métaphore. Il parait légitime de s'interroger sur la capacité de cette figure du discours à produire la violence de l'expérience comme événement du texte, dans la mesure où, événement elle-même par l'écart qu'elle ouvre entre la norme de la langue et la singularité innovante de la parole, elle fait «violence au réel» par «l'équivalence imaginative » qu'elle impose, pour reprendre les termes de Paul Ricœur ${ }^{2}$. Cependant l'impact qu'elle génère ne prend pas corps dans le texte sous la forme d'un bouleversement syntaxique, car, comme l'analyse Jean-Jacques Lecercle dans La Violence du langage, il ne s'agit pas d'enfreindre les règles « essentielles » c'est-à-dire syntaxiques, mais seulement de jouer un niveau d'interprétation contre un autre, ce qui «n'entraîne pas le même degré de bouleversement dans la langue que la violation des règles syntaxiques $»^{3}$.

3 L'objet de cette étude sera donc de tenter de déterminer d'où provient l'effet de violence induit par l'utilisation originale que Crane a faite de la métaphore dans son roman de 
guerre dans le dessein de mettre en relief, pour mieux le dénoncer, le choc des armes au moyen du choc des mots. C'est le conflit des éléments sémantiques issu du processus de condensation qui retiendra toute notre attention, puisque la métaphore se fonde sur l'incompatibilité sémique, et nous verrons comment une exploitation radicale de cette caractéristique centrale est productrice de tension. En outre, l'opposition antithétique des signifiants se fait le véhicule de l'horreur quand elle allie le domaine festif au tragique de la mort dans une veine carnavalesque. Cette transgression des limites conventionnelles, qui se retrouve dans la confusion des catégories servant habituellement à la perception du moi et du monde, traduit la perte de sens de l'individu menacé par un réel qu'il ne maîtrise pas. Dès lors, la métaphore se présente comme l'inscription dans le texte de l'horreur du sujet qui ne se reconnaît pas dans l'expérience physiquement aliénante de la guerre.

4 L'intention de Crane dans The Red Badge of Courage est de faire voir la guerre autrement que dans les récits de guerre des décennies qui l'ont précédée et que dans le discours impérialiste de l'époque. Pour bousculer les habitudes de pensée de ses lecteurs, il procède à coups de petits électrochocs linguistiques dont la métaphore s'avère l'instrument privilégié, en ce qu'elle établit un écart entre le sens propre d'un signifiant et un sens figuré insolite. Que le romancier compte sur l'effet de violence produit par l'insolite se manifeste dans son recours répété, dans les descriptions de scènes de batailles, à la métaphore ornementale qui dérange, une métaphore visiblement insérée en vue de créer un effet et non dans un but de clarification sémantique. L'objectif est d'attirer l'attention sur l'écart ainsi ouvert à partir de la norme de représentation et de signaler la métaphore comme le véhicule d'un sens autre.

5 Pour faire advenir dans le discours la violence qu'il entreprend de dénoncer, Crane constitue la métaphore en un lieu de tension, créant par là «l'effet esthétique » que Laurent Jenny définit dans La Parole singulière comme «le jeu de tensions » que le texte «met en œuvre pour provoquer le lecteur à la construction du sens $»^{4}$. On peut dégager deux sources de tension dans la métaphore de The Red Badge, celle qui provient du jeu entre la littéralité et la figuralité, et celle que produit l'incompatibilité sémique. Dans le premier cas, Crane crée un choc plus grand en faisant se télescoper les deux niveaux - littéral et figuré - que la figure met en relation, en les maintenant également présents dans l'énoncé au moyen d'un retour au référentiel.

6 Ce procédé est perceptible en particulier dans son travail de redynamisation de la métaphore éteinte, par lequel il engendre un effet de « bougé » en réactivant la littéralité oubliée. Si les expressions courantes «colère noire » ou « rouge de colère » ne suscitent plus aucune surprise, en revanche la « rage livide » du héros Henry Fleming impuissant face à l'agonie terrifiante de son ami Jim Conklin, souligne la violence dévastatrice de l'événement observé, en donnant un sens littéral à l'adjectif de couleur véhicule de l'analogie et en lui faisant refléter l'effroi effectif du personnage ${ }^{5}$. En sollicitant la sensation visuelle ancrée dans le réel plutôt qu'un objet absent, la figure acquiert plus d'impact.

7 Une autre illustration de ce principe est fournie dans la périphrase «the flaming opposition» désignant le tir de barrage de l'armée ennemie (79). Les flammes métaphoriques consacrées par l'usage pour décrire une animation, voire animosité, verbale, retrouvent ici leur sens premier réactualisé par le contexte des armes à feu, et de ce fait l'opposition en question n'en devient que plus palpable et percutante. Le choc provient de la prise de conscience que l'agression n'est pas une abstraction éloignée, une 
analogie théorique, mais un donné de l'expérience menaçant directement le sujet, à l'image de ce qu'effectue l'auteur à l'échelle du roman en centrant ses préoccupations sur le vécu subjectif du soldat individuel. La violence à décrire se trouve introduite dans le discours sous la forme d'une irruption du réel au sein du figural.

La même tension anime les métaphores vives de l'œuvre. Ainsi, au lieu de substituer un objet absent à l'élément considéré afin de dégager des caractéristiques par ressemblance, le discours narratif emprunte l'analogie à un objet issu du champ référentiel, ne procédant de ce fait pas radicalement à la rupture d'isotopie que Ricœur place au cœur de la définition de la métaphore traditionnelle ${ }^{6}$. L'effet de surprise provient de ce que le sème commun est dérivé non pas de l'expérience ordinaire mais de celle de la guerre. La présence de celle-ci se trouve ainsi accrue dans la représentation puisque s'ouvre à elle un champ qu'elle n'occupe pas habituellement. Par exemple, la rage d'un lieutenant face à un régiment récalcitrant sous le feu des balles adverses est évoquée dans les termes suivants : " the remainder of his idea disappeared in a blue haze of curses » (79). C'est ici la fumée régnant sur le champ de bataille qui fournit l'image, autrement dit un objet référentiel qui devient métaphorique par un retournement original. Le mot « haze », outil de l'analogie, a la double tâche de dénoter le référentiel auquel il est censé se substituer et connoter un sens figuré absent. C'est ce court-circuit entre deux exigences opposées qui induit la tension perceptible dans l'énoncé.

9 Le même phénomène en sens contraire est à l'œuvre dans la phrase : "One window, glowing a deep murder red, shone squarely through the leaves » (74). Il s'opère ici une inversion des rôles entre terme propre et terme métaphorique. Le meurtre dont la couleur rouge est ordinairement la métaphore grâce au sème commun du sang, devient lui-même métaphore de la couleur prise dans son sens dénotatif. Le mot « rouge » émerge alors comme un foyer de tension entre les deux fonctions, résultant de l'instabilité de la langue, et cette tension permet d'exprimer toute la violence d'une situation où domine le rouge du sang. La présence de «murder » dans l'évocation de la fenêtre fait implicitement surgir la notion de sang, sème commun à "rouge " et à "meurtre ", qui vient ternir l'objet décrit, et rappelle la source d'horreur du champ de bataille. La métaphore du meurtre a pour effet de sur-déterminer de violence l'élément du décor avant même que celui-ci ne soit posé.

Crane exploite également toutes les ressources tensionnelles de l'incompatibilité sémique de la métaphore, qui, selon Ricœur, distingue cette figure de la comparaison-similitude ${ }^{7}$. C'est le choc de la «collision sémantique » qui attire l'attention sur l'enjeu de la scène représentée. Avant d'aborder cet aspect proprement dit, il serait bon de souligner un type d'incohérence particulier présent dans une métaphore récurrente du roman, que l'on pourrait qualifier de synesthésique. Le texte abonde en "black curses ", " red cheers ", " crimson roar » $(85,38,37)$ et autres manifestations colorées de la fougue belliqueuse. La figure de la métaphore se double ici d'un autre trope, la synesthésie, par laquelle le plus souvent Crane rend le fracas insupportable de la guerre en s'appuyant sur la couleur. L'intensité de violence que ne peut adéquatement exprimer un adjectif quantitatif ordinaire, fût-il hyperbolique, comme "formidable ", est mise en relief par l'écart entre deux domaines sensoriels distincts. Le surplus de sens vient se loger dans le hiatus ouvert à la surface du texte entre deux sensations d'ordre différent puis résonne de la force conjuguée des deux connotations, emplissant le vide créé d'un soudain excès. L'amplification, l'exagération induite par la synesthésie contribue à la surdétermination 
de sens de la métaphore, que Jean-Jacques Lecercle note à propos du jeu de mots ou de l'allitération'.

11 D'une manière générale, l'équation prononcée entre des éléments a priori disparates est source de tension. L'impossibilité référentielle constitue le signe d'un événement contrenature dans l'ordre du réel que le sujet ne veut pas reconnaître. Ainsi la fusillade ennemie qui menace le héros est transcrite par le non-sens suivant au niveau littéral : « it seemed that all forward ways were barred by the thin leaping tongues»(79). De façon significative, l'impossibilité linguistique est associée à une situation d'impasse sur le plan narratif, un piège qui se referme sur le personnage, l'expérience de la violence extrême. À la perplexité immédiate engendrée par l'image incongrue de langues qui bondissent, un incohérent mélange de concret et d'abstrait, vient s'ajouter la confusion causée par le télescopage de multiples connotations issues de l'intertextualité intra et extradiégétiques : le langage des armes - une métaphore récurrente du roman - la cacophonie des langues de la Tour de Babel, la suggestion ironique de la langue de feu de la Pentecôte qui combine l'impression visuelle de la flamme et l'image figurative du discours. Cet excès de sens fait violence à l'esprit du lecteur, mais au départ c'est l'incompatibilité sémique qui retient l'attention et signale l'intensité accrue.

ressemblance, que Ricœur place au cœur du processus métaphorique plutôt que la perception de la différence, devient une opération de violence, la conciliation de l'inconciliable. Ce phénomène est souvent exprimé par le recours à l'allitération, qui par sa surcharge sonore met en relief l'insolite association de signifiants discordants tout en amorçant la fusion par la production de l'identique : " the sobs scorched him », «the grim, stalking specter of a soldier » (42), « four or five corpses keeping mournful company » (38). L'allitération sert ainsi à exprimer matériellement le choc abstrait de concepts antithétiques que Crane provoque par le biais de la métaphore, dans une radicalisation de la figure qui lui est propre.

13 Par la «collision sémantique » qu'elle engendre, la métaphore permet le rapprochement de domaines aussi distants et ordinairement jugés incompatibles que la réjouissance et le deuil, le rire et la mort. Car l'expérience centrale de The Red Badge of Courage est bien la mort que le jeune protagoniste doit apprendre à regarder en face au cours de son initiation sur le champ de bataille. La violence de l'émotion générée à chacune des scènes successives de confrontation et de familiarisation avec le grand Ennemi n'est pas exprimée de manière explicite, mais reflétée par la brutalité du langage, le choc des mots, et en particulier le recours au champ lexical du festif pour véhiculer l'analogie.

Un passage significatif à cet égard est la scène qui clôt le chapitre IX, à savoir le trépas du vaillant soldat Jim Conklin, ami de Henry. Au cours de cet épisode burlesque qui raille la vision héroïque de la mort colportée par la littérature traditionnelle du genre, Crane apparente le violent tremblement marquant l'agonie du personnage à une danse : « For a moment the tremor of his legs caused him to dance a sort of hideous hornpipe » (44). Le sème commun de mouvement frénétique, qui apparaît dans sa version négative dans l'élément référentiel « tremor », est valorisé positivement dans le terme métaphorique de «dance ». L'agitation incontrôlée, le désordre du réel, sont évacués au profit du mouvement harmonieux et maîtrisé connoté par l'analogie, le tragique de la mort imminente remplacé par la gaieté. La métaphore nous introduit ainsi dans le monde carnavalesque marqué par l'ambivalence. Le complément d'objet reproduit la dualité en contredisant l'expression de la réjouissance collective que constitue la danse de la matelote au moyen de l'adjectif subjectif « hideous » marquant l'extrême répulsion. Le 
hiatus sémantique entre les deux est comblé par l'identité sonore de l'allitération en [h], expulsion matérielle de l'horreur entrevue. La phrase suivante poursuit dans la même veine : «His arms beat wildly about his head in expression of implike enthusiasm ». Un glissement s'effectue du désordre menaçant suggéré par l'adverbe "wildly à la réjouissance véhiculée par le substantif final via le personnage de l'analogie, le lutin, sympathique euphémisation du diable qui oscille de manière ambivalente entre le positif et le négatif. Notons au passage que la même association d'idées réapparaît dans une description d'attaque vers la fin du roman, lorsque les soldats couverts de poussière et s'agitant en tous sens sont assimilés à des démons dansant la gigue : "they were, with their swaying bodies, black faces, and glowing eyes, like strange and ugly fiends jigging heavily in the smoke » (90).

L'élément festif introduit par la métaphore débouche à la fin de l'épisode sur le thème du rire, complètement discordant dans le contexte: «The mouth was open and the teeth showed in a laugh », image qui n'est pas sans évoquer les représentations médiévales grimaçantes de la mort. La violence de l'énoncé tient à ce miroir déformant de la métaphore qui montre une image inversée de la réalité et ne semble pouvoir traduire le choc de la scène qu'en renvoyant à son contraire plus conforme au désir, en proposant de ce fait une antithèse si monstrueuse que l'horreur indicible du référent se trouve réintroduite dans le décalage narratif. La violence est ainsi déplacée de l'événement raconté à l'événement linguistique.

16 La métaphore de Crane participe de ce fait du grotesque, et s'inscrit dans la veine clairement affichée de cette version anti-héroïque de la mort du bon soldat. Complétant la thématique de la déformation qui tout au long de la scène exerce une violence contrenature sur la ligne droite associée à la dignité du corps vivant, la métaphore rejoint le grotesque en ce qu'elle tente d'unir des éléments que tout sépare. Dans son ouvrage intitulé On the Grotesque, Geoffrey Harpham définit le genre en des termes qui pourraient s'appliquer à la métaphore : «the sense that things that should be kept apart are fused together ", ce qui l'amène en conclusion de son analyse à suggérer une analogie entre les deux procédés : «Both metaphor and grotesque give a dominant impression of unity, though they are manifestly constructed of pieces. And both operate by means of selfabolishing incongruity ${ }^{9}$. Le rapprochement incongru est dans les deux cas à l'origine de l'effet de violence.

Outre la danse et le rire, Crane fait appel à des images empruntées aux domaines de la musique et du jeu, et substitue la notion de gaieté à la souffrance dominante. Une illustration frappante en est donnée dans un passage du chapitre VIII qui retrace le poignant repli d'un groupe de blessés. L'audace de l'évocation paraît dérangeante au point que le discours n'ose franchir le pas, et préfère rester prudemment du côté de la comparaison-similitude, comme si l'identification opérée par la figure de la métaphore constituait une transgression frisant le sacrilège: "One of the wounded men had a shoeful of blood. He hopped like a schoolboy in a game. He was laughing hysterically. [...] One was marching with an air imitative of some sublime drum major. [...] As he marched he sang a bit of doggerel [...] » (38). Toute la constellation est ici présente : le jeu, le rire, la musique. L'effet de violence naît de la juxtaposition contre-nature de l'horreur et de la réjouissance, comme le souligne le texte lui-même à propos de l'expression du visage d'un blessé, « an unholy mixture of merriment and agony ». Cet énoncé pourrait parfaitement caractériser la métaphore de Crane et l'on notera l'adjectif révélateur qui met l'accent sur le caractère « impie » du rapprochement. 
18 De même dans le chapitre XXII, la véritable boucherie en cours sur le champ de bataille entraîne la vision suivante des drapeaux témoins de la folie meurtrière des hommes: "The youth could see the two flags shaking with laughter amid the smoke remnants » (89).

19 L'animisme permet d'attribuer à des objets une attitude trop intolérable pour être associée à un être humain. Le rire face à semblable spectacle constitue tout ce qu'il y a de plus choquant et nous renvoie à l'analyse du carnavalesque par Mikhail Bakhtine, moyen de combattre la peur cosmique par le rire. La représentation de la guerre selon le mode ironique de Crane n'est pas sans rappeler l'explication que le critique russe fournit à propos de la bastonnade : «Les images visent à englober les deux pôles du devenir dans leur unité contradictoire. Le battu (ou tué) est paré ; la bastonnade est joyeuse [...] au milieu des rires $»^{10}$. Ce qui nous intéresse ici est la cohabitation du rire et de la mutilation corporelle, l'antithèse exprimée en un même mouvement, une même perception de l'esprit, union inattendue des contraires, opérée par la métaphore dans The Red Badge of Courage.

20 L'image du spectacle, récurrente dans le roman pour qualifier les scènes de violence, peut également se lire dans cette perspective. Loin d'être évitées, ces scènes sont données à voir aux personnages diégétiques et sont érigées en spectacle. Si la mort de Jim Conklin revêt des aspects de "cérémonie » rituelle ("The youth had watched, spellbound, this ceremony at the place of meeting »,44), la décimation du régiment du héros prend franchement des allures de représentation théâtrale: «He was deeply absorbed as a spectator. The crash and swing of the great drama made him lean forward» (90) ou encore " The men saw a ground vacant of fighters. It would have been an empty stage if it were not for a few corpses » (84). Le caractère carnavalesque de réjouissance devient explicite dans la suite de la dernière citation : " At sight of this tableau, » (autre forme du donné à voir) « many of the men in blue sprang from behind their covers and made an ungainly dance of joy " qui nous ramène à notre précédent développement. La valorisation $d u$ motif $d u$ spectacle apparaît enfin dans la fascination qu'il exerce. L'adjectif «spellbound » mentionné plus haut est appliqué au héros confronté à son ami agonisant, tandis que les soldats contemplent ainsi le massacre de leur régiment: "This spectacle seemed to paralyze them, overcome them with a fatal fascination » (78), l'allitération en [f] accusant une fois de plus le paradoxe. L'analyse de Mikhaïl Bakhtine sur la fonction du spectacle de carnaval apporte un éclairage intéressant à l'image telle qu'elle est utilisée par Crane: "Le carnaval remporte une victoire définitive et transforme les enfers en un joyeux spectacle juste bon à être montré sur la place publique », une manifestation selon lui de « la peur vaincue par le rire ${ }^{11}$. Autrement dit, la métaphore carnavalesque qui introduit une dimension festive dans l'univers terrifiant de la mort, tente d'exorciser l'horreur du champ de bataille.

21 Par-delà la collision de pôles antithétiques, la violence de la métaphore dans le roman provient de son élément de transgression observé par Jean-François Lyotard : «La vraie métaphore, le trope, commence avec l'excès dans l'écart, avec la transgression du champ des substituables reçus par l'usage $»^{12}$. Jean-Jacques Lecercle note également le «scandale» engendré par la métaphore dans sa caractéristique d'exagération «lorsqu'une frontière est franchie $»^{13}$. C'est aussi sous cet angle que l'on peut analyser le recours de Crane au carnavalesque pour évoquer les souffrances du soldat blessé ou agonisant, car le rire et la gaieté n'apparaissent-ils pas comme une transgression dans le monde solennel de la mort? La substitution du comique au pathétique bafoue la norme 
morale conventionnelle. Mais c'est la violence issue de la transgression de frontière entre champs sémantiques qui nous occupe ici, et c'est par elle qu'est le mieux traduit le pathos du corps mutilé.

En effet, la perception du corps humain en dehors des catégories qui lui sont propres et lui confèrent son identité, est source d'angoisse pour le sujet qui ne se reconnaît plus dans son être et se sent menacé d'annihilation. La violence de la guerre se trouve ainsi exprimée dans l'aliénation opérée par cette confusion linguistique au sein de la métaphore. On peut distinguer deux types majeurs de confusion inacceptables pour l'esprit, le brouillage entre l'animé et l'inanimé, puis l'indistinction entre l'individu et le groupe, le moi et l'autre. Tout d'abord, le corps n'est plus perçu comme doté de la vie qui le distingue des objets inanimés dont il se sert, alors qu'inversement la vie semble continuer dans le corps frappé par la mort. La violence résulte de la réification du soldat, $\mathrm{du}$ fait que le corps se voit infliger le traitement habituellement réservé aux choses inanimées, et par là la spécificité de l'être humain être niée : «from this position [the enemy] began briskly to slice up the blue men » (91).

Cette inquiétante opération de saucissonnage méthodique trouve son écho dans un exemple voisin, qui développe les suggestions éveillées par la comparaison de la bataille à une immense machine, dans une veine toute conradienne ${ }^{14}:$ "The battle was like the grinding of an immense and terrible machine to him. [...] He must go close and see it produce corpses » (38). Le choc provient de la représentation des victimes comme le produit d'une fabrication industrielle, taylorisation monstrueuse d'un événement singulier intolérable. La métaphore permet l'invasion de l'humain par l'inanimé, par l'inhumain, et du contingent par la répétition. Le corps du soldat est traité avec la même désinvolture que s'il s'agissait d'un objet insignifiant, et l'extrême violence de l'événement unique que constitue sa mort se voit occultée de façon scandaleuse par le pluriel et la banalité, comme l'illustre cet autre exemple : «it looked as if they would merely succeed in making a great sprinkling of corpses on the grass » (92). Le recours au gérondif qui traduit une vue globalisante accentue la perte d'identité individuelle résultant de la réification.

24 À l'inverse, la métaphore suggère l'inacceptable de la mort en affirmant, contre toute vraisemblance référentielle par une sorte d'illusion de la perception, la persistance de la vie dans la mort. L'enseigne ennemi à qui Henry veut arracher le symbole de la victoire, se cramponne à son drapeau après avoir trépassé, comme si c'était à la vie qu'il ne voulait pas renoncer. Il reste le sujet actif du discours narratif et présente toutes les caractéristiques d'un vivant: «the corpse swayed forward with bowed head. One arm swung high, and the curved hand fell with heavy protest on the friend's unheeding shoulder » (80). De même, dans l'épisode de face-à-face avec un cadavre au cœur de la forêt, le texte procède à un inquiétant échange d'attributs entre Henry et le soldat mort érigé en double potentiel du héros à travers l'importante thématique du regard qui régit la rencontre : « He was being looked at by a dead man [...] He was for moments turned to stone before it. [...] The dead man and the living man exchanged a long look » (36).

Un deuxième type de confusion brutale est effectué grâce à la conjonction de la métaphore et d'une figure de la contiguïté, la métonymie, qui par le glissement qu'elle opère, efface la limite entre l'individu et le groupe. Le régiment est en effet représenté de manière persistante comme un grand corps subissant les mutilations de la guerre, à l'instar du soldat particulier: "a blood-stained crowd streaming to the rear ", "the steady current of the maimed»(38). La double exagération résultant de la métonymie 
alliée à la métaphore aquatique accroît le pathos du corps blessé en attribuant à l'effusion de sang une échelle gigantesque. L'effet de violence dérive de cette extension démesurée de la blessure qui décuple l'action destructrice de la guerre et hypertrophie le phénomène. Mais il tient aussi à la déshumanisation conséquente à la vision collective, à la négation de l'individu fondu dans la masse. La souffrance personnelle se trouve banalisée par la répétition et le pluriel, accentués dans le dernier exemple par l'adjectif « steady » qui vient renforcer les suggestions du « courant ». L'individu se voit finalement dépossédé de ce qui fait l'unicité de sa propre expérience et apparaît dès lors indifférencié de l'autre.

C'est bien en dernier ressort la troublante vision simultanée du même et de l'autre que véhicule la métaphore. L'incompatibilité sémique des contraires traduit la violence du choc produit par la désorientation du moi qui ne se reconnait pas, par la perception de l'étranger dans le familier, cette inquiétante étrangeté que Freud a analysée dans le thème du double. De fait, l'horreur éprouvée par Henry face au corps de son ami déréglé par l'agonie, et exprimée dans le texte narratif par le télescopage du pathétique et du festif au sein de la métaphore de la danse, s'accompagne d'un sentiment révélateur de malaise: "there was a curious and profound dignity in the firm lines of his awful face » (44). Les adjectifs « curious » et « awful » soulignent la surprise et l'horreur du sujet qui ne reconnait pas l'objet familier, cet alter ego que constitue le frère d'armes ami défiguré par l'ombre de la mort. La phrase qui suit explicite la situation: «He was invaded by a creeping strangeness that slowly enveloped him » et c'est à cet instant précis que s'inscrit dans le texte la métaphore de la danse, indice du flux de violence interne. C'est le spectacle de sa propre mort que le héros refuse de reconnaitre dans cette scène, tout comme dans celle, antérieure, de rencontre à caractère autoscopique avec un cadavre anonyme dans la forêt.

Les frontières tombent, les limites rassurantes disparaissent, laissant la place au mouvant et au discontinu. Au lieu de la rigidité attendue, les rangs de l'armée présentent la sinuosité du serpent, avec toute l'horreur attachée à l'animal mythique, ou celle de la vague qui menace d'engloutissement, enregistrant par là les soubresauts de l'angoisse de l'observateur : " He expected a battle scene ", on attend alors une bataille bien rangée, mais la réalité est tout autre: «There were some little fields girted and squeezed by a forest. [...] he could see knots and waving lines of skirmishers »(18). Le summum est atteint lorsque les deux aspects sont combinés au sein d'un même énoncé et se constituent en paradoxe grinçant: "the steady current of the maimed»(38). Non seulement le sujet est aux prises avec la vision simultanée des deux sources d'angoisse, le mouvant et l'incomplétude, mais il se dégage en outre une incompatibilité inconciliable entre le dynamisme du premier et la privation d'énergie du second.

Confusion de l'animé et de l'inanimé, du moi et de l'autre, la confusion linguistique établie par la métaphore pour rendre compte de la violence du champ de bataille, s'avère en fait signaler l'effondrement des limites qui se produit lors de la vision de ce que Julia Kristeva définit comme l'abject. L'expérience de la guerre n'est autre que la confrontation avec l'horreur de la mort, le cadavre grotesque, cette expression ultime de l'abjection. Julia Kristeva décrit en effet le cadavre biblique comme un "élément trouble entre l'animé et l'inorganique, grouillement de transition ", et par là, " un corps sans âme, un non-corps, une matière trouble, il est à exclure du territoire comme de la parole de Dieu $»^{15}$. De la même façon, l'abject entrevu dans les combats est à exclure du discours narratif et est donc déplacé par la figure de la métaphore, qui fait un détour par un autre signifiant 
de façon à tenir à distance le fait dérangeant, tout en signalant la violence de celui-ci par la collision sémantique. La violence du rejet se traduit par l'écart figural, tandis que le choc causé par la condensation linguistique constitue la trace de l'événement. Dans de nombreux cas, la surdétermination de la métaphore vient renforcer l'effet produit et compenser l'absence par l'excès sémantique. Le phénomène est parfaitement illustré par la suite donnée, au début du chapitre $\mathrm{X}$, à l'expiration de Jim Conklin. Face à l'horreur de la situation, le héros est frappé d'aphasie, et la mort qu'il ne veut pas admettre est transportée dans le domaine du discours : « The youth desired to screech out his grief. He was stabbed, but his tongue lay dead in the tomb of his mouth » (44). La figure souligne ici en même temps l'incapacité à dire la violence vécue et la nécessité de dévier celle-ci. La métaphore, redoublée dans ce cas précis par les prolongements métonymiques de l'image de la bouche qui se substitue au corps, permet ainsi d'enregistrer l'événement dans le discours, sans le transcrire en tant que tel dans toute son insupportable réalité. Comme dans le mécanisme du rêve, elle donne la possibilité d'exprimer ce dont l'esprit se détourne en l'euphémisant en quelque sorte, seul mode acceptable du dire.

L'effondrement des catégories lié à la perception de l'abject qu'est la mort, entraîne la disparition des certitudes et laisse place à un monde où plus aucune immunité n'est garantie, où les zones traditionnelles de sécurité sont ébranlées et où le même est potentiellement son contraire. La métaphore de Crane remet en question des notions bien assises dans leur légitimité en les compromettant avec leurs opposés, en brouillant valorisation positive et négative. Par exemple, l'innocence habituellement attribuée à l'enfance est ternie quand elle est associée à la violence des jurons d'un officier incapable de maîtriser la déroute de son régiment : « He strode suddenly forth, his infantile features black with rage » (78). Le télescopage des adjectifs « infantile » et «black » qui encadrent le substantif, met en relief la contamination d'un domaine ordinairement du côté de la pureté avec sa métaphore de blancheur, par la noirceur de la fureur destructrice. Le même genre de processus est à l'œuvre dans « he could string oaths with the facility of a maiden who strings beads» (78). Cet ébranlement des certitudes par le brouillage des catégories établies confère à l'écriture de Crane une dimension moderniste.

Cependant cette entreprise reste timide, et il convient de souligner, pour terminer, les contradictions perceptibles dans l'utilisation de la métaphore par Crane. Celle-ci apparaît comme une figure audacieuse à laquelle l'auteur ne s'abandonne pas complètement. Il demeure encore très dépendant de la comparaison-similitude dans son répertoire analogique, préférant souvent garder les deux sémantismes antithétiques distincts dans des éléments séparés plutôt que de les fondre dans une même identité trop déstabilisante. Il est révélateur à cet égard de constater qu'à diverses reprises, le discours passe d'abord par la comparaison avant de procéder au choc de la métaphore, comme pour préparer l'esprit du lecteur à la violence de l'énoncé final. C'est le cas de l'image obsédante de l'œil qui domine les chapitres d'ouverture, appliquée aux feux de camp de l'armée ennemie. Nous avons aussi eu l'occasion d'en citer d'autres comme: "The battle was like the grinding of an immense and terrible machine. [...] He must go close and see it produce corpses ». Ou inversement, le discours fait une brève incursion dans la métaphore pour se replier sur la comparaison: " he could string oaths with the facility of a maiden who strings beads » ou "His arms beat wildly about his head in expression of implike enthusiasm » qui succède à la métaphore de la danse macabre. Le texte revient ainsi à la forme plus conventionnelle de deux énoncés demeurant distincts le long de deux lignes parallèles. 
De la même façon, on remarque une utilisation paradoxale de la métaphore chez Crane, partagée entre la volonté de dire la violence de la guerre en accordant une place plus grande au référentiel, et la tendance inverse à s'en détourner, quand l'horreur devient indicible, en pratiquant l'écart rendu possible par la figure. Sans doute ce paradoxe est-il imputable au dessein du romancier de choquer les consciences sans pour autant être suffisamment dégagé de la tradition et des conventions dénoncées. Ce dilemme parait inscrit dans la démarche foncièrement ironique de l'œuvre, qui implique une écriture très contrôlée et détachée de son objet, une retenue constante. On se heurte à chaque pas à une ironie omniprésente, souvent proche du sarcasme, qui maintient tout à distance et fait du processus analogique lui-même une opération délibérée interdisant le jeu de mécanismes plus irrationnels, une façon de se protéger d'un sujet explosif. La violence imprimée à l'œuvre est contenue. Affirmée d'un côté avec la véhémence de l'iconoclaste, elle est dans le même temps limitée par peur ou incapacité d'aller trop loin, à la fois vis-àvis de la tradition littéraire et dans l'affrontement des démons intérieurs.

\section{BIBLIOGRAPHIE}

Bakhtine, Mikhail. L'Ëuvre de François Rabelais et la culture au Moyen-Age et sous la Renaissance. Paris : NRF Gallimard, 1970.

Bergon, F. Stephen Crane's Artistry. New York: Columbia UP, 1975.

Cox, J.T. "The Imagery of The Red Badge of Courage”. Modern Fiction Studies, 5, 1959, 209-219.

Fontanier, P. Les Figures du discours. Paris : Flammarion, 1968.

Harpham, G. On the Grotesque : Strategies of Contradiction in Art and Literature. Princeton: Princeton UP, 1982.

Jenny, Laurent. La Parole singulière. Paris : Belin, 1990.

Karl, F.R. et Lawrence Davies (eds.). The Collected Letters of Joseph Conrad., vol. I, 1861-1895.

Cambridge/London/New York: Cambridge UP, 1983.

Knapp, B. Stephen Crane. New York: The Ungar Publishing Company, 1987.

Kristeva, Julia. Pouvoirs de l'horreur. Paris : Éditions du Seuil, 1980.

Lecercle, Jean-Jacques. La Violence du langage. Paris : PUF, 1996.

Lyotard, Jean-François. Discours, figure. Paris : Klincksieck, 1985.

Morier, H. Dictionnaire de poétique et de rhétorique. Paris : PUF, 1961.

Pizer, D. Realism and Naturalism in Nineteenth Century American Literature. Carbondale: Southern Illinois UP, 1984.

Ricœur , Paul. La Métaphore vive. Paris : Éditions du Seuil, 1975.

Wogan, C.C. "Crane's Use of Colour in The Red Badge of Courage". Modern Fiction Studies, 6, 1960, 168-172. 


\section{NOTES}

1. "You are a complete impressionist. The illusions of life come out of your hand without a flaw. " Lettre de Joseph Conrad à Stephen Crane en date de décembre 1897, http:// xroads.virginia.edu/HYPER/CRANE/reviews/conrad.html

2. Paul Ricœur, La Métaphore vive, Paris, Éditions du Seuil, 1975 : «L'équivalence imaginative instaurée par la métaphore fait plus violence au réel que la métonymie », 255.

3. Jean-Jacques Lecercle, La Violence du langage, Paris, PUF, 1996, 153.

4. Laurent Jenny, La Parole singulière, Paris, Belin, 1990, 83, note de bas de page 29.

5. « The youth turned, with sudden, livid rage, toward the battlefield. " Stephen Crane, The Red Badge of Courage, 1895, New York/London, W.W. Norton \& Company, 1994, 44. Toutes les références ultérieures au roman seront tirées de cette édition.

6. Ricœur souligne «la nécessité d'incorporer la rupture d'isotopie à la définition même de la métaphore " qu'il particularise comme un «écart par rapport au contexte». Paul Ricœur, La Métaphore vive, op. cit., 32.

7. Ibid., 236: «[...] dans la métaphore, la perception d'une incompatibilité est essentielle à l'interprétation du message ». Cette incompatibilité est aussi qualifiée plus loin de "collision sémantique ", 246.

8. Ibid., 158.

9. Geoffrey Galt Harpham, « On the Grotesque : Strategies of Contradiction » in Art and Literature, Princeton, New Jersey, Princeton UP, 1982, n 11, 178.

10. Mikhail Bakhtine, L'Euvre de François Rabelais et la culture au Moyen-Age et sous la Renaissance, Paris, NRF Gallimard, 1970, 204.

11. Ibid., 391.

12. Jean-François Lyotard, Discours, Figure, Paris, Klincksieck, 1985, 254-255.

13. Jean-Jacques Lecercle, La Violence du langage, op. cit., 173.

14. Cette image évoque la déclaration, devenue célèbre, de Joseph Conrad dans une lettre à Cunningham Grahame : "There is a way-clet us say-a machine. It evolved itself (I am severely scientific) out of a chaos of scraps of iron and behold!-it knits. I am horrified at the horrible work and stand appalled. [...] It knits us in and it knits us out. It has knitted time, space, pain, death, corruption, despair and all the illusions-and nothing matters. ", 20 décembre 1897, Frederick R. Karl and Lawrence Davies ed., The Collected Letters of Joseph Conrad, vol. I, 1861-1895, Cambridge/London/New York, Cambridge UP, 1983, 425.

15. Julia Kristeva, Pouvoirs de l'horreur, Paris, Éditions du Seuil, 1980, 127.

\section{INDEX}

oeuvrecitee Red Badge of Courage (The) 